\title{
SPECIES-POOL HYPOTHESIS: LIMITS TO ITS TESTING
}

\author{
Jan Lepš \\ Department of Botany, Faculty of Biological Sciences, University of South Bohemia and Institute of \\ Entomology, Academy of Sciences of the Czech Republic, Branišovská 31, CZ-370 05 České Budějovice, \\ Czech Republic; e-mail suspa@tix.bf.jcu.cz
}

Keywords: Competition, Null model, Species diversity

\begin{abstract}
The species richness of a community depends both on the pool of available species and on biotic mechanisms that lead to the exclusion of some of the species from a community. The method suggested by PÄRTEL et al., Oikos 75: 111-117, 1996 to test the effect of species-pool size on the species richness of a community is discussed in this paper. This method is based on the calculation of a correlation between the actual species richness of a community and the actual species-pool size, and compares the correlation found in the data with that simulated by a null model. In the null model, the species richness has a uniform distribution between zero and the size of the species pool. A correlation significantly higher than that in the null model is interpreted as evidence of the greater role of species pool than of local interactions in formation of community species richness (ZOBEL, Folia Geobot. 36: 3-8, 2001). It is shown that the interpretation of discrepancies between the null model and reality is difficult or impossible, because: (1) a null model with a uniform distribution of species richness is unrealistic, (2) both models based on the random selection of species from a species pool and models that include competitive interactions in the community predict a higher positive correlation of species richness and size of the species pool than the null model, and (3) local species richness might be affected by species-pool size, but a large species-pool size can also be a result of high local species richness. Caution is urged when interpreting the analyses based on the size of the filtered species-pool size.
\end{abstract}

\section{INTRODUCTION}

The species richness of a community depends on both the pool of available species (the pool of species able to grow under given environmental conditions that can migrate to a particular site) and biotic mechanisms that lead to the exclusion of some of the species from a community (TAYLOR et al. 1990, ZOBEL 1997). The biotic mechanism most often operating in terrestrial plant communities among vascular plants is probably competition. Both species-pool size and competition operate simultaneously in determining species composition and are not mutually exclusive. The dependence of local species richness on the species pool appears to be obvious (in any case, the number of species in a community cannot exceed the number of species in the species pool). However, if a community becomes "saturated" by species, i.e., limitation by competition is very strong, and the number of species in the species pool is much higher than local species richness, then the relationship between local richness and species-pool size may be weak. The dependence of local species richness on the size of the species pool over a range of large species-pool sizes may be weak - the relationship exhibits a richness ceiling (CORNELL 1993).

PÄRTEL et al. (1996) and ZOBEL (2001) suggested and advocated a method of testing the effect of species-pool size on local richness: because species richness cannot exceed the size 
of the species pool, the correlation (expressed as the correlation coefficient, $r$ ) between the size of an actual species pool and the actual local species richness is compared with the correlation in a null model (quasi-neutral model in ZOBEL 2001). In the null model, local species richness has a uniform distribution, i.e., all values between zero and the size of the species pool are equally likely. A higher correlation in the actual data than in the null model signifies the effect of the species pool. The facts that the correlation is usually much higher than predicted by the null model and that species richness is usually a relatively constant proportion of the species pool are used by ZOBEL (2001) as part of a body of evidence for his conclusion that competition does not play an important role in the formation of plant communities.

I see three problems with this approach. The first is the formulation of the null model, the second is the interpretation of possible differences between the null model and real data, and the third is a problem of delimiting the species pool. The goal of this paper is to analyze these problems.

In this paper, I will use the term species pool for the set of species available on a larger spatial scale, and (local) species richness for the species actually present within a plot under consideration. Generally, this reasoning can be applied to any relationship between species richness on smaller and larger scales, i.e., between local and regional species pools or between actual and local species pools sensu ZoBEL (1997).

\section{THE NULL MODELS}

In the formulation of the null model of PÄrTEL et al. (1996) and ZoBEL (2001) a uniform distribution of species richness (between zero and the species pool size) is used. This is an extremely unlikely situation. I am not able to invent any feasible mechanisms leading to such a distribution. Theoretically a very tricky mechanism might be constructed, however, it would necessarily include many positive associations between species - a uniform distribution cannot be achieved when species independence is expected. Values close to zero and values close to the size of the species pool are extremely unlikely. These states can only be achieved when the absence of one species decreases the probability of the presence of other species and the presence of a species increases the probability of the presence of other species with all the dependencies carefully balanced. Such a mechanism is extremely unlikely; so the null model does not satisfy one of the basics of null models, i.e., simplicity. Refutation of a complicated null model is hardly evidence for a mechanism.

In contrast, there is a simple model that is based on species independence. When each species has the same probability $(p)$ of being included in a community (the proportional model of CORNELL 1993) then community species richness has a binomial distribution with a mean $=p . n$ and variance $=n p(1-p)$. This null model is more flexible than the uniform distribution model because the average ratio between local species richness and the size of the species pool can be incorporated in the model through the $p$ value. For $p=0.5$ the model simulates the same average species richness as the uniform distribution but the variance is much smaller (for $n>3$ ). For the binomial distribution variance is $n / 4$, whereas for the uniform distribution the variance can be approximated by $n^{2} / 12$. Because of this the correlation between local richness and species pool-size is much higher for the binomial distribution.

To demonstrate this I have simulated communities with species richness having a uniform distribution and having a binomial distribution for a species pool of 5 to 100 species with 100 replications for each species-pool size (Fig. 1). Whereas the correlation coefficient between 
species-pool size and local richness (calculated from all 2000 simulated communities) was 0.97 for the binomial distribution, it was only 0.63 for the uniform distribution. The null model, which does not include any species interactions, predicts a higher correlation than the model with uniform species distribution.

\section{COMPETITION MODELS ALSO PREDICT THE POSITIVE EFFECT OF SPECIES POOL ON LOCAL SPECIES RICHNESS}

Even when species composition is controlled by competition, local species richness will most likely be correlated with the size of the species pool. To examine this, I have simulated the competition dynamics of communities using a set of Lotka-Volterra equations of the form:

$$
\frac{\mathrm{d} n_{i}}{\mathrm{~d} t}=r_{i} N_{i}\left\{\frac{K_{i}-\sum_{j} \alpha_{i j} N_{j}}{K_{i}}\right\}
$$

where $N_{i}$ is the population size of $i$-th species, $r_{i}$ and $K_{i}$ are growth rate and carrying capacity of the $i$-th species, respectively, and $\alpha_{i j}$ are competition coefficients. $\alpha_{i i}=1$ for all the species. In all my simulations, the $K$ and $r$ values were the same for all the species $(K=100, r=0.15)$, and the competition coefficients $\left(\alpha_{i j}\right.$ for $\left.i \neq j\right)$ were generated randomly. Two sets were generated, one with weak competition ( $\alpha_{i j}$ drawn from a uniform distribution between 0 and 1) and one with strong competition (uniform distribution between 0 and 2). For each species-pool size, 100 independent matrices of competition coefficients were generated under "strong competition" and 100 under "weak competition". The initial population size of each species was $K / s, s$ being the number of species in the pool. The models were simulated till time $t=500$, which was sufficient to reach equilibrium, and the number of species surviving till equilibrium (technically, species with $N_{i}>0.01$ ) were counted. The correlation between the number of species at equilibrium and species-pool size was 0.85 and 0.67 for the weak and strong competition matrices, respectively (each for the 2000 communities, see Fig. 1). Both correlations are higher than the correlation for the uniform distribution of species counts, but lower than the correlation for the binomial distribution (i.e., for the assumption of species independence). For the strong competition matrix, the correlation is lower than for the weak competition, and close to that of uniform distribution. Even when strong competition was simulated up to a pool size of 250 species (not shown here), there was no clear tendency towards saturation (ceiling). Nevertheless, the existence of a ceiling clearly depends on the formulation of the model; as noted by CoRNELL (1993), "nearly as many models predict no saturation as predict saturation, and saturation may be absent even in strongly interactive communities".

Summarizing this part, we can conclude:

(1) There are no feasible mechanisms that would generate a uniform distribution of local richness (i.e., all species richness values between zero and the number of species in the species pool are equally likely to appear);

(2) Feasible models (both including and excluding interspecific competition) lead to a stronger correlation between local richness and species-pool size than the model based on the assumption of a uniform distribution of local species richness. 

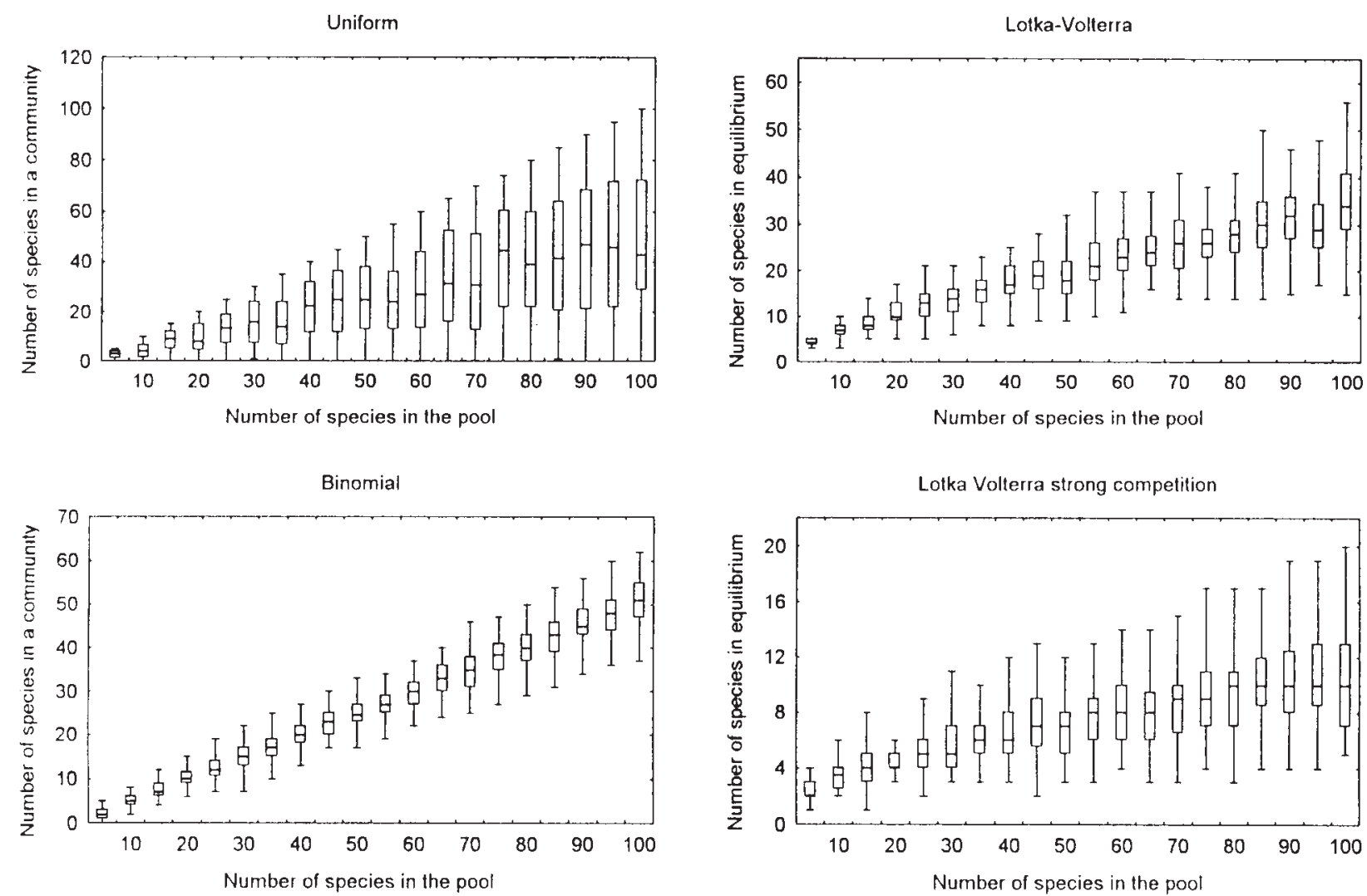

Fig. 1. Number of species in a community as a function of the size of the species pool, as simulated by a model with uniform distribution of species richness, bimodal distribution of species richness and Lotka-Volterra models with weak and strong competition. Each box and whisker is based on 100 simulations. Whiskers extend to the extremes, box corresponds to the interquartile range with median.

Consequently, finding a higher correlation between local species richness and species-pool size in real data than that found in the uniformly-distributed species richness model does not indicate anything.

If a binomial distribution were taken as the null model, then a correlation smaller than predicted (or a tendency towards saturation) might be considered as an indication of mechanisms causing species in a community to not be random selections from the species pool; this mechanism is most likely being competition. However, a better test than the one based on the comparison of correlation coefficients would be one based on the shape of the relationship; if there is a saturation effect then the function of dependence of local richness on species-pool size should be concave. This would probably be best demonstrated by showing that $b_{2}$ is significantly negative in the regression:

$$
\text { Local richness }=b_{0}+b_{1} \text {. Pool }+b_{2} \text { Pool }^{2}
$$

(see CORNELL 1993). Negative results (i.e., $b_{2}$ is not significantly different from zero) should be interpreted with extreme caution, because the acceptance of the null hypothesis $b_{2}=0$ is a decision with an unknown rate of Type II error (we do not know the power of the test), particularly when the sample size is small.

However, the effect of competition has been demonstrated many times experimentally (AARSSEN \& EPP 1990), even for plants of similar height. For example, Gentiana pneumonanthe became extinct in many meadows after the cessation of mowing, because it was outcompeted, 
often by tussock grasses of similar height. The mechanism need not be direct shading; rather the seedling recruitment of gentians is hindered by the dense turf (KŘENOVÁ \& LEPŠ 1996) and populations became extinct due to demographic senescence.

When using the null model approach all of the cautionary notes on the application of null models have to be applied (LEPŠ 1990: violation of any of the assumptions of the null model can lead to a discrepancy between model predictions and data). One should also be aware that the absence of a ceiling does not show that the communities are non-interactive. This is because even some models that include competition do not predict saturation (for example, $b_{2}$ was not significantly different from zero in the L-V simulations) and for those which predict a ceiling it is extremely difficult to say at what pool size the ceiling would start to be detectable.

\section{WHAT IS THE CAUSE AND WHAT IS THE CONSEQUENCE?}

A further cautionary note should be added: the above models consider the species pool to be an independent variable, the causal path being: species pool $\rightarrow$ local species richness. However, as shown by HERBEN (2000), a large species pool may follow from many species being able to coexist locally and thus, the large size of the species pool is a consequence of this high local species richness (i.e., species are able to avoid competitive exclusion). On the other hand, high local species richness may be a consequence of large species pool (typical chicken and egg problem, see also CORNELl 1993). For example, in Central Europe more species are able to grow in oligotrophic meadows than in highly eutrophic meadows (i.e., the oligotrophic meadow species pool is larger). In addition, the local species richness of oligotrophic meadows is higher. There is no way to deduce from these facts whether the species pool of oligotrophic meadows is large because, under conditions of low productivity, species are able to avoid competitive exclusion and high local species richness results in a large species pool, or whether (e.g., for historical reasons) fewer species are adapted to high-nutrient environments. It is generally believed that it is competition for light that drives species to extinction when oligotrophic meadows are fertilized. This is because, with a decrease in nutrient limitation, light competition becomes decisive and competition for light is more asymmetric than competition for nutrients (ZOBEL 1992, LEPŠ 1999). It is also true that the number of species able to compete in high-nutrient environments is limited. Even if we know that the species that are dominant in low-nutrient environments become even more dominant with increased nutrients and that this leads to the exclusion of competitively inferior species (as in LEPŠ 1999), we cannot exclude the possibility that the decrease in species richness occurs because there were no species available (i.e., able to invade) that would be able to compete under high-nutrient conditions. For most environmentally-driven changes in species richness we can show (e.g., experimentally) that the proximate cause is the change in competitive relationships, but we cannot exclude the possibility that the ultimate cause is the change in the size of the species pool.

A species pool, at any spatial scale, is affected by evolutionary processes, immigration and extinction. At least the latter two are affected by competition. For example, in the postglacial era new invaders often spread into established communities and thus the rate of spread was regulated by competition, and similarly, the species that became extinct during postglacial times were usually outcompeted, e.g., by taller vegetation invading during favourable climatic periods (TALLIS 1991). Consequently, the size of the species pool is affected by competition (past or present). 


\section{DEFINITIONS OF SPECIES POOL: FILTERED AND UNFILTERED}

The species pool can be defined in two ways. Neither definition is incorrect, however, the interpretation of results depends on how the terms are defined. We can either define the species pool as (1) "a set of species which are potentially capable of coexisting in a certain community" (ERIKSSON 1993), or (2) as a set of species which are able to grow under the given abiotic conditions. In ZoBEL's (1997) terminology, the species list is either filtered (1) or not (2). The two definitions differ in whether the effect of biotic factors are or are not included. Each of the definitions has its advantages and its appeal, but also its limitations.

In particular, for the first definition (filtered species list), if the species are able to coexist then they are able to avoid competitive exclusion. So it should be, as per the definition, impossible to find examples of competitive exclusion there. That is, the species from the species pool that are absent in an actual plot are missing for other reasons than competitive exclusion. This applies to some extent to all the methods of determination of the species pool as described in ZoBEL et al. (1998). For example, when using Ellenberg's indicator values (ELLENBERG et al. 1991), species with low indicator values for nitrogen would be excluded from the species pool for eutrophic meadows. However, those species have low indicator values for nitrogen essentially because they are driven to extinction in eutrophic meadows by competition. Consequently, the conclusions that "selection from regional species pool into actual species pool, and selection from actual species pool into microsite are mostly random and neutral processes and they are not directed significantly by interspecific competition" (ZOBEL 2001) is an inevitable consequence of the definition of species pool.

On the other hand, it would be interesting to know how the species pool for a particular community depends, for example, on its habitat durability. However, in all investigations it has to be kept in mind that species pool size also depends on the area of corresponding habitat according to species-area relationships. This relationship holds, whether we estimate the size of the species pool directly by a survey of some area, or whether we estimate it by scanning a flora. For example, in the flora of the Czech Republic there is a limited number of species of alpine calcareous rocks; this is a consequence of the fact that the area of alpine calcareous rocks is extremely limited in the Czech Republic.

Nearly all plant species found in Central Europe immigrated after glacial retreat. Consequently, the size of the filtered species pool will depend on migration rates, and this in turn, may be (negatively) correlated with competition. One of the reasons for this might be the trade-off between competitive ability and dispersability (SOUTHWOOD 1988), demonstrated, e.g., during early stages of succession on Mount St. Helens (TITUS \& DEL MoRAL 1998). The filtered species pool is affected by local processes, such as competition, distance of the sources of the species, their dispersability, and the possible proliferation of species from other community types.

The "unfiltered" definition of the species pool has the advantage that it does not confound abiotic limitations with the effect of biotic interactions. It can easily be shown experimentally that many species are able to reach the site, to grow under the prevailing abiotic conditions (thus belonging to the unfiltered species pool), but are eliminated from the community by competition. However, ZOBEL (1997) argued that "it makes no sense to compare number of species in grassland community with that in a region that includes seashore and raised bog as well as grassland". I do not think that this is so irrelevant. In similar but distant landscapes, the differences in the number of species in a region reflect mostly historical factors. Thus, it 
is useful for comparisons between regions, not for comparison of community types within a region.

\section{THERE ARE NO SIMPLE SOLUTIONS}

I think that there is little debate as to whether the species richness of a plant community is influenced by both the species pool and local processes. (This is in contrast to some invertebrate herbivore assemblages, where the effects of local interactions are seriously doubted, e.g., LAWTON et al. 1993). However, it is of interest to know how much of the variability in community species richness can be explained by differences between corresponding species pools, and how much by local processes. I have found the approach that studies "convergence", i.e., the constancy of relationships of species richness in various habitats over geographic regions to be very convincing (SCHLUTER \& RICKLEFS 1993). This analytical approach is based on the ideas of two-way analysis of variance. The total variability in species richness is split into the part that can be explained by regional differences and the part that can be explained by differences between habitats. I do not see feasible possibilities for de-coupling the effects of local processes (competition) from the effects of the species pool for particular habitats within a region. This is because the formation of the latter is affected by the former. Positive correlations between local species richness and (filtered) species-pool size of corresponding community types is expected under the various models and consequently cannot be used as evidence for any of the models.

Acknowledgement: I wish to express my gratitude to Kristjan Zobel for writing a thought-provoking paper, which successfully stimulated discussion. I thank Tomáš Herben and Jonathan Titus for helpful comments, and Jonathan Titus for linguistic help. Research was supported by the GAČR grant 206/99/0889.

\section{REFERENCES}

AARSSEN L. \& EPP G.A. (1990): Neighbour manipulations in a natural vegetation: a review. J. Veg. Sci. 1: $13-30$.

CORNELL H.V. (1993): Unsaturated patterns in species assemblages: the role of regional processes in setting local species richness. In: RICKLEFS R.E. \& SCHLUTER D. (eds.), Species diversity in ecological communities, Historical and geographical perspectives, The University of Chicago Press, Chicago, pp. 243-252.

Ellenberg H., Weber H.E., Düll R., Wirth V., Werner W. \& PAulissen D. (1991): Zeigenwerte von Pflanzen in Mitteleuropa. Scripta Geobot. 18: 1-248.

ERIKSSON O. (1993): The species-pool hypothesis and plant community diversity. Oikos 68: 371-374.

HERBEN T. (2000): Correlation between richness per unit area and the species pool cannot be used to demonstrate the species pool effect. J.Veg. Sci. 11: 123-126.

Ǩ̌ENOVÁ Z. \& LEPŠ J. (1996): Regeneration of a Gentiana pneumonanthe population in an oligotrophic wet meadow. J. Veg. Sci. 7: 107-112.

LAWTON J.H., LEWINSOHN T.M. \& COMPTON S.G. (1993): Patterns of diversity for the insect herbivores on bracken. In: RICKLEFS R.E. \& SCHLUTER D. (eds.), Species diversity in ecological communities, Historical and geographical perspectives, The University of Chicago Press, Chicago, pp. 178-184.

LEPŠ J. (1990): Can underlying mechanisms be deduced from observed patterns? In: KRAHULEC F., AGNEW A.D.Q., AGnew S. \& WILlEMS J.H. (eds.), Spatial processes in plant communities, SPB Publ., The Hague, pp. $1-11$.

LEPŠ J. (1999): Nutrient status, disturbance and competition: an experimental test of relationships in a wet meadow. J. Veg. Sci. 10: 219-230.

PÄRTEL M., ZOBEL M., ZOBEL K. \& VAN DER MAAREL E. (1996): The species pool and its relation to species richness: evidence from Estonian plant communities. Oikos 75: 111-117. 
SCHLUTER D. \& RICKLEFS R.E. (1993): Convergence and regional components of species diversity. In: RICKLEFS R.E. \& SCHLUTER D. (eds.), Species diversity in ecological communities, Historical and geographical perspectives, The University of Chicago Press, Chicago, pp. 230-240.

SouTHWOOD T.R.E. (1988): Tactics, strategies and templets. Oikos 52: 3-18.

TALLIS J.H. (1991): Plant community history. Chapman and Hall, London.

TAYLORD.R., AARSSEN L.W. \& LOEHLEC. (1990): On the relationship between r/K selection and environmental carrying capacity: a new habitat templet for plant life history strategies. Oikos 58: 239-350.

TITUS J. \& DEL MORAL R. (1998): Seedling establishment in different microsites on Mount St. Helens, Washington, USA. Pl. Ecol. 134: 13-26.

ZOBEL M. (1997): The relative role of species pools in determining plant species richness: an alternative explanation of species coexistence? Trends Ecol. Evol. 12: 266-269.

ZOBEL K. (2001): On the species-pool hypothesis and on the quasi-neutral concept of plant community diversity. Folia Geobot. 36: 3-8.

ZOBEL M. (1992): Plant species coexistence the role of historical, evolutionary and ecological factors. Oikos 65: 314-320.

Zobel M., VAN DeR MAAREL E. \& Dupré C. (1998): Species pool: the concept, its determination and significance for community restoration. Appl. Veg. Sci. 1: 55-66. 